\title{
SPIN-PHONON MAGNETIC RESONANCE OF CONDUCTION ELECTRONS IN INDIUM ANTIMONIDE CRYSTALS
}

\author{
N. A. Poklonski, ${ }^{*}$ A. N. Dzeraviaha, and S. A. Vyrko
}

UDC 621.315 .592

Resonance absorption of radio waves (with a frequency of $10 \mathrm{MHz}$ ) by c-band electrons in indium antimonide crystals doped with hydrogen-like donors (tellurium atoms) at room temperature in an external magnetic field is theoretically studied. Known experimental data obtained for samples with electron concentrations in the range from $6 \cdot 10^{15}$ to $5 \cdot 10^{18} \mathrm{~cm}^{-3}$ are analyzed and interpreted. Resonant absorption of radio waves by $n$-InSb:Te crystals in a magnetic field is calculated to be due to spin-phonon resonance based on the law of conservation of energy and the quasi-wave vector for electrons and optical phonons. The resonance arises as a result of a spin-flip interaction of a c-band electron with an optical phonon, which is assisted by resonant absorption of radio waves in a magnetic field. A physical picture of the phenomenon is given. Analytical relations are presented. Calculations are carried out and are consistent with experimental data that could not previously be interpreted at all.

Keywords: spin-phonon magnetic resonance, spin flip, electron, optical phonon, absorption of radio waves.

Introduction. Crystalline layers of $n$-type InSb are used in spintronics devices [1] owing to the anomalous negative effective magnetic moment of the $c$-band electrons $\left(\left|\mu_{n}\right| \approx 26 \mu_{\mathrm{B}}\right.$, where $\mu_{\mathrm{B}}$ is the Bohr magneton) [2]. The $c$-band electrons also have much greater drift mobility (under laboratory conditions) than those of $\mathrm{Si}, \mathrm{Ge}$, and diamond crystals. This enables fast-acting semiconducting instruments based on $n$-InSb to be fabricated. For example, a model for a high-frequency field transistor of InSb nanowires was proposed [3]. The above features are a consequence of undoped InSb crystals having a very small density of states $c$-band electron effective mass $\left(m=0.0136 m_{0}[4,5]\right.$, where $m_{0}$ is the electron mass in a vacuum). However, the effective mass $m$ increases considerably upon increasing the concentration of free electrons (conductivity electrons) as a result of doping InSb with hydrogen-like donors [6]. Furthermore, InSb crystals are direct-bandgap narrowbandgap semiconductors [7]. The bandgap of $E_{\mathrm{g}} \approx 0.18 \mathrm{eV}$ at room temperature is much less than the electron affinity $\mathrm{EA} \approx 4.6 \mathrm{eV}[8]$. The narrow bandgap between the bottom of the $c$-band and the top of the $v$-band complicates calculations of the one-electron band structure of InSb as compared to, e.g., analogous calculations for diamond and Si because strong spinorbit coupling of the $c$-band electrons with heavy and light $v$-band holes and holes in the spin-orbit split off $v$-band subband must be taken into account $[7,9]$. Hall sensors, which are widely used to measure magnetic field strength, were prepared from InSb crystals [10]. All this is responsible for the practical importance of studying the effects of elemental dopants and external magnetic fields on electronic states and processes in $n$-InSb.

Magnetic resonance in six $n$-InSb:Te crystals was studied over 30 years ago by Kondrat'ev [11, 12]. Measurements were made on a nuclear magnetic resonance spectrometer (henceforth radiospectrometer) at operating RF $\omega_{\mathrm{rw}} / 2 \pi=10 \mathrm{MHz}$ at magnetic field strength $B<1.7 \mathrm{~T}$ and field frequency modulation $70 \mathrm{~Hz}$. The positions of the resonances were practically independent of temperature. Their intensities allowed measurements to be made at room temperature (Fig. 1). The samples $(\sim 1.5 \times 2 \times 10 \mathrm{~mm})$ were situated with the long side $(10 \mathrm{~mm})$ along the coil axis of the radiospectrometer resonance cavity. The minimal dimension $(1.5 \mathrm{~mm})$ of the $n$-InSb:Te samples corresponded to the [211] crystal growth direction. The first derivative $Y^{\prime}=\mathrm{d} Q / \mathrm{d} B$ of the quality factor $Q$ of cavity loaded with sample was recorded as a function of the scanning magnetic field strength $B$. The center of the resonance corresponded to $Y^{\prime}=0$ for $B=B_{\mathrm{r}}$. The concentrations of $c$-band electrons in samples Nos. $1-6$ at $77 \mathrm{~K}$ were $n=6 \cdot 10^{15}, 1 \cdot 10^{16}, 5.8 \cdot 10^{16}, 1 \cdot 10^{17}, 9 \cdot 10^{17}$, and $5 \cdot 10^{18} \mathrm{~cm}^{-3}$, respectively. The Te atom in the InSb crystalline matrix replaced an $\mathrm{Sb}$ atom and was a hydrogen-like donor. The thermal ionization energy of Te atoms in

* To whom correspondence should be addressed.

Belarusian State University, 220030, Minsk, Belarus; email: poklonski@bsu.by. Translated from Zhurnal Prikladnoi Spektroskopii, Vol. 87, No. 4, pp. 595-604, July-August, 2020. Original article submitted June 5, 2020. 

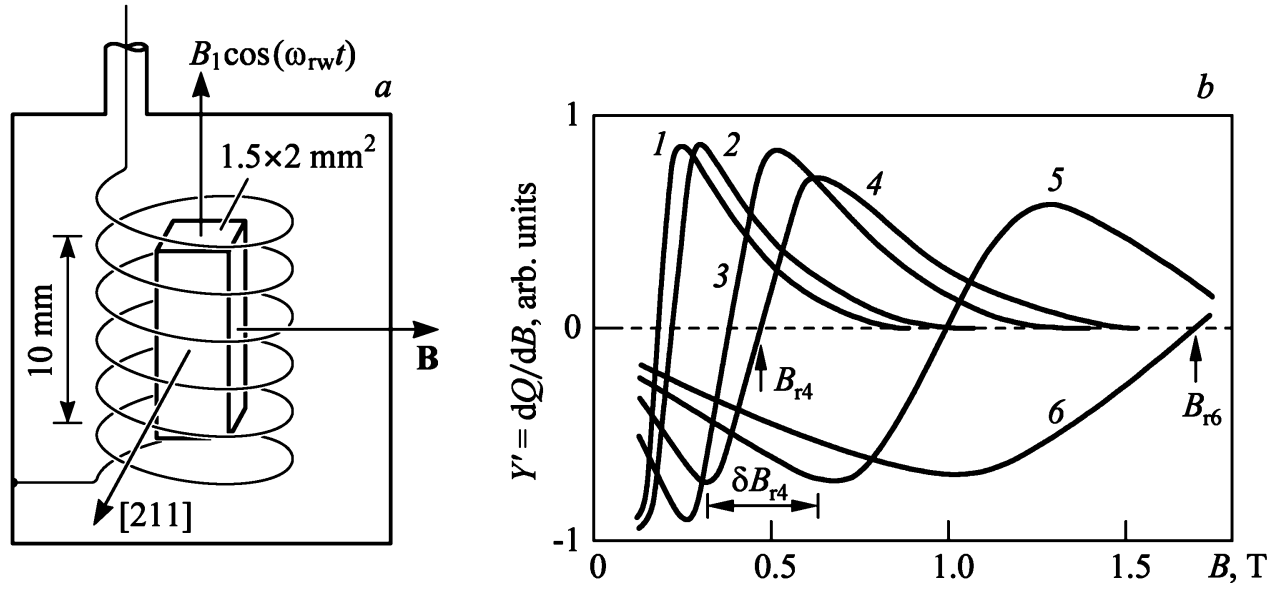

Fig. 1. Experimental setup: $\mathbf{B} \perp[211]$ is the scanning constant magnetic field in the sample; $B_{1} \cos \left(\omega_{\mathrm{rw}} t\right)$ is the variable magnetic field in the resonator coil (a); magnetic resonance signals $Y^{\prime}=\mathrm{d} Q / \mathrm{d} B$ of $n$-InSb:Te samples at $300 \mathrm{~K}$ for electron concentrations $6 \cdot 10^{15}(1)$, $1 \cdot 10^{16}(2), 5.8 \cdot 10^{16}(3), 1 \cdot 10^{17}(4), 9 \cdot 10^{17}(5)$, and $5 \cdot 10^{18} \mathrm{~cm}^{-3}(6)$; constant frequency $f_{\mathrm{rw}}=\omega_{\mathrm{rw}} / 2 \pi=10 \mathrm{MHz}$ of electromagnetic field of radiospectrometer coil; figures adapted from the literature [12] (b).

the limiting weakly doped samples was $\sim 0.7 \mathrm{meV}$. The degree of compensation (concentration ratio of acceptors, all in the -1 charge state, to Te atoms) was $K \approx 0.1$ in all samples. The Te atoms at room temperature were fully ionized (i.e., had the +1 charge state). Therefore, samples Nos. 3-6 were located on the metallic side of the insulator-metal concentration phase transition [13].

At liquid He temperature $(4.2 \mathrm{~K})$, the high-field tail of the resonance of sample No. 4 had Shubnikov-de Haas oscillations [11] related to quantization of $c$-band electron density of states in strong magnetic fields. They were observed at low temperatures and resulted from overlap of Landau and Fermi levels [14]. The oscillations disappeared and the resonance weakened as the temperature was increased to $300 \mathrm{~K}$. However, the resonant magnetic strength $B_{\mathrm{r}}$ practically did not change [11]. The width $\delta B_{\mathrm{r}}$ of the resonance line increased (from $\delta B_{\mathrm{r}} \approx 0.13 \mathrm{~T}$ for $n=6 \cdot 10^{15} \mathrm{~cm}^{-3}$ to $\delta B_{\mathrm{r}} \approx 1.34 \mathrm{~T}$ for $n=5 \cdot 10^{18} \mathrm{~cm}^{-3}$ ) as the concentration of $c$-band electrons increased. However, the tails of the magnetic resonance line became more symmetric. It was also found that the centers of resonance lines determined from the condition $Y^{\prime}\left(B_{\mathrm{r}}\right)=0$ (Fig. 1b) obeyed the equation $B_{\mathrm{r}}=\alpha f_{\mathrm{rw}} n^{1 / 3}\left[1-\beta\left(3 \cos ^{2} \varphi-1\right)\right]$, where $\alpha=0.97 \cdot 10^{-13} \mathrm{~T} \cdot \mathrm{cm} \cdot \mathrm{s} ; f_{\mathrm{rw}}=\omega_{\mathrm{rw}} / 2 \pi=10 \mathrm{MHz}$ is the radiospectrometer cavity resonance frequency; $\beta=0.1$; and $\varphi$, the angle between the direction $B$ of the constant magnetic field and the crystallographic [211] direction in the sample [12]. The center $B_{\mathrm{r}}$, width $\delta B_{\mathrm{r}}$, and integrated intensity of lines $Y$ (i.e., the integral of $\left|Y^{\prime}(B)\right|$ over magnetic field strength from 0 to $3 B_{\mathrm{r}}$ ) had approximately the same dependence on the electron concentration $n$ with $\delta B_{\mathrm{r}} \approx B_{\mathrm{r}}$. The position of the center of resonance line $B_{\mathrm{r}}$ depended on the angle between the direction of the magnetic field and the [211] direction in the crystals because of anisotropy of the $c$-band electron $g$-factor $[15,16]$.

Hypotheses. Interpretation of the measured resonant absorption of radio waves of frequency $f_{\mathrm{rw}}=10 \mathrm{MHz}$ by $n$-InSb:Te crystals in a magnetic field led to proposed and refuted hypotheses that the depth of the skin-layer increased in a magnetic field; ultrasound generated by the measuring radiospectrometer coil was absorbed by conductivity electrons and/or ${ }^{115}$ In and ${ }^{121} \mathrm{Sb}$ nuclei of the InSb crystal matrix; and magnetostriction appeared in the samples [17].

The goal of the present work was to propose a model that described quantitatively the magnetic resonance measurements in $n$-InSb:Te crystals at room temperature [11, 12].

Let us also discuss hypotheses that were not considered before [11, 12] regarding various types of magnetic resonances [18] in solids: 1) ultrasound absorption by ${ }^{125} \mathrm{Te}$ nuclei [the hypothesis was refuted because the position of the center of the magnetic resonance varied in the range $0.1-1 \mathrm{mT}$ while the line width (from peak to peak of the first derivative of the resonance line vs. the magnetic field) varied by $\sim 1 \mathrm{mT}$ [19], which did not agree with the values given in Table 1]; 2) a Knight shift $\Delta B_{\mathrm{K}}$ of the center of the $B_{\mathrm{nmr}}$ line for resonant absorption of radio waves by atomic nuclei magnetic moments because of the additional magnetic field imposed on them by $c$-band electrons (the hypothesis was refuted because of the small shift of the NMR lines $\Delta B_{\mathrm{K}} / B_{\mathrm{nmr}} \approx 2 \cdot 10^{-4}$ [20] depending on the concentration of $c$-band electrons as compared to the data in Fig. 1b); 3) cyclotron resonance of the $c$-band electrons $\hbar \omega_{\mathrm{rw}}=\hbar \omega_{\mathrm{c}}$, where $\hbar=h / 2 \pi$ is Planck's constant; $\omega_{\mathrm{rw}}=2 \pi f_{\mathrm{rw}}$, 
TABLE 1. Experimental [12] Parameters of Magnetic Resonance Signals in $n$-Type InSb Crystals as Functions of Electron Concentration

\begin{tabular}{|c|c|c|c|c|c|c|c|}
\hline$j$ & $n, \mathrm{~cm}^{-3}$ & $\sigma_{\mathrm{dc}},(\Omega \cdot \mathrm{cm})^{-1}$ & $\delta_{\mathrm{s}}, \mathrm{mm}$ & $B_{\mathrm{r}}^{(\mathrm{e})}, \mathrm{T}$ & $B_{\mathrm{r}}^{(\mathrm{t})}, \mathrm{T}$ & $\delta B_{\mathrm{r}}^{(\mathrm{e})}, \mathrm{T}$ & $Y_{j}^{(\mathrm{e})} / Y_{1}^{(\mathrm{e})}$ \\
\hline 1 & $6 \cdot 10^{15}$ & 78.17 & 1.8 & 0.18 & 0.18 & 0.13 & 1 \\
2 & $1 \cdot 10^{16}$ & 125.4 & 1.4 & 0.22 & 0.23 & 0.17 & 1.5 \\
3 & $5.8 \cdot 10^{16}$ & 563.4 & 0.67 & 0.38 & 0.38 & 0.25 & 3.4 \\
4 & $1 \cdot 10^{17}$ & 880.5 & 0.55 & 0.47 & 0.45 & 0.31 & 3.9 \\
5 & $9 \cdot 10^{17}$ & 3233 & 0.28 & 0.99 & 0.93 & 0.61 & 10 \\
6 & $5 \cdot 10^{18}$ & 6897 & 0.19 & 1.70 & 1.60 & 1.34 & 40 \\
\hline
\end{tabular}

Note. Values of $\sigma_{\mathrm{dc}}=e n \mu(n)$ from Eq. (6) and $\delta_{\mathrm{s}}$ from Eq. (11) were calculated for zero magnetic field; resonance magnetic strength (experimental $B_{\mathrm{r}}^{(\mathrm{e})}$ and $B_{\mathrm{r}}^{(\mathrm{t})}$ calculated from Eq. (4) considering Eq. (16) for absorption of optical phonons in the center of the Brillouin zone); magnetic resonance signal width $\delta B_{\mathrm{r}}^{(\mathrm{e})}$ and intensity ratio $Y_{j}^{(\mathrm{e})} / Y_{1}^{(\mathrm{e})}$ of the signal for the $j$ th sample to the first sample.

the angular frequency of the electromagnetic field created by the cavity coil in the studied sample; $\omega_{\mathrm{c}}=e B / m$, the angular cyclotron frequency; $e$, the elementary charge; $B$, the external magnetic field strength; and $m$, the $c$-band electron effective mass (the hypothesis was refuted because the condition for observation of cyclotron resonance was not fulfilled because $\left.\omega_{\mathrm{c}}>>\omega_{\mathrm{rw}}[21]\right)$; 4) spin resonance in conductivity electrons: $\hbar \omega_{\mathrm{rw}}=\left|g_{n}\right| \mu_{\mathrm{B}} B_{\mathrm{r}}$, where $\left|g_{n}\right| \approx 51$ is the $g$-factor of a $c$-band electron; $\mu_{\mathrm{B}}=e \hbar / 2 m_{0}$, the Bohr magnetron (the hypothesis was refuted because the condition for resonant absorption was fulfilled for magnetic field strength $B_{\mathrm{r}} \approx 10 \mu \mathrm{T}$ ); 5) spin-phonon resonance: transition of a $c$-band electron between Zeeman sublevels of Landau levels in a magnetic field upon absorption by the electron of an optical phonon. This transition led to a change of direction of the electron spin relative to the direction of the scanning magnetic field induction and absorption of radio waves $\left(f_{\mathrm{rw}}=10 \mathrm{MHz}\right)$. The dependence of the $c$-band electron $g$-factor on its quasi-wave vector was responsible for the coupling of the optical phonon and electron spin [22, 23].

General Relationships. Let us discuss hypothesis 5. The effects of optical phonons responsible for magnetic resonance signals, i.e., cyclotron-phonon [24], spin-cyclotron-phonon [25], and spin-magnetophonon resonances [26-28], were considered before. The sound absorption coefficient associated with transfer of a $c$-band electron between Landau levels and simultaneous inversion of its spin were calculated [25]. A model for spin inversion because of coupling of a $c$-band electron with the lattice but without a change in the number $\zeta$ of the Landau parabola (levels) was given [28] (Fig. 2). Also, magnetophonon resonance, i.e., transfer of a $c$-band electron between Landau levels upon absorption of an optical phonon without spin inversion is known $[29,30]$. It is noteworthy that localization of $c$-band electrons on donor ions in an external magnetic field [31, 32] was not considered in this work because the magnetic resonance in InSb crystals was measured at room temperature, i.e., with full thermal ionization of the Te atoms.

The motion of the electrons becomes unidimensional in a rather strong external magnetic field $\left[\omega_{\mathrm{c}} \tau>1\right.$, where $\tau$ is the average relaxation time of the $c$-band electron (quasi)wave vector $\mathbf{k}]$. They can move with kinetic energy $(\hbar \mathbf{k})^{2} / 2 m$ only along or against the direction of the external magnetic field vector $\mathbf{B}$, i.e., $\mathbf{k} \| \mathbf{B}$. The magnetic field does not act on phonons so that they can move in any direction.

Let us examine phonon absorption by a $c$-band electron with wave vector projection $k_{1 z}$ on the $z$ axis parallel to the external field vector with subsequent spin inversion and a transition of the electron into a Landau level corresponding to a higher energy of electron. In this instance, the law of conservation of energy for spin-phonon magnetic resonance is written:

$$
\frac{\left(\hbar k_{2 z}\right)^{2}}{2 m}+\left|g_{n}\right| \mu_{\mathrm{B}} B_{\mathrm{r}}+\left(\zeta_{\mathrm{f}}-\zeta_{\mathrm{i}}\right) \hbar \omega_{\mathrm{c}}=\frac{\left(\hbar k_{1 z}\right)^{2}}{2 m}+E_{\mathrm{ph}}(q)+\hbar \omega_{\mathrm{rw}},
$$

where $k_{2 z}$ is the $z$-component of the $c$-band electron wave vector [after absorption of a phonon with energy $E_{\mathrm{ph}}(q)$ and (quasi)wave vector value $q] ;\left|g_{n}\right| \mu_{\mathrm{B}} B_{\mathrm{r}}$, Zeeman splitting of each Landau level; $\omega_{\mathrm{c}}$, angular cyclotron frequency at resonance $\left(B=B_{\mathrm{r}}\right) ; \zeta_{\mathrm{i}}$ and $\zeta_{\mathrm{f}}$, Landau level numbers before and after phonon absorption; and $\hbar \omega_{\mathrm{rw}}$, a quantum of radio-wave signal energy (detecting the resonance) with angular frequency $\omega_{\mathrm{rw}}$. It is noteworthy that electron transitions occur primarily from the most populated Landau levels $\left(\zeta_{\mathrm{i}}=0\right.$ and $\left.\zeta_{\mathrm{i}}=1\right)$ into the final Landau level $\zeta_{\mathrm{f}}>\zeta_{\mathrm{i}}$. Figure 2 shows a diagram of electron 


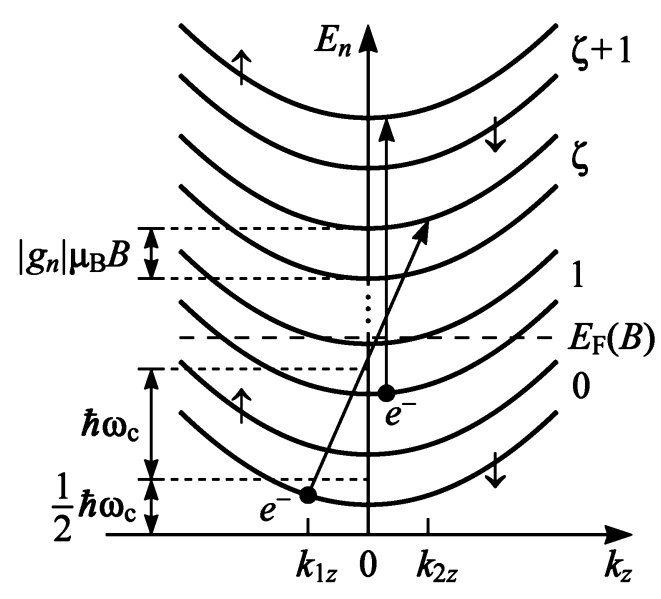

Fig. 2. Diagram of Landau levels (parabolas) and Zeeman sublevels of $c$-band electrons of $n$-InSb:Te crystals in an external constant magnetic field; $E_{n}$ is the single-electron energy, $k_{2}$, electron wave vector component along the magnetic strength vector; $\hbar \omega_{\mathrm{c}}$, energy of cyclotron splitting of electron energy levels, $\left|g_{n}\right| \mu_{\mathrm{B}} B$, Zeeman splitting energy; arrow shows spin-phonon transition of $c$-band electron $\left(e^{-}\right) ; E_{\mathrm{F}}(B)$, Fermi level depending on magnetic field strength $B ; \zeta=0,1, \ldots$ correspond to Landau level number; arrows denote directions of electron spin along $(\uparrow)$ and against $(\downarrow)$ the direction of magnetic field strength for $g_{n}<0$.

transitions between Zeeman sublevels of Landau levels (parabolas) with spin inversion. The arrows show direct and indirect electron transitions on the single-electron energy-wave vector diagram.

The law of conservation of $c$-band electron wave vector $z$-component imposes a condition in addition to Eq. (1) on the value of the wave vectors of phonons involved in producing the magnetic resonance signal:

$$
k_{2 z}=k_{1 z}+q \cos \theta \text {, }
$$

where $q$ is the phonon wave vector modulus; $\theta$, the angle between the directions of the phonon wave vector $\mathbf{q}$ in the crystal and the $z$ axis. By substituting Eq. (2) into Eq. (1), we obtain:

$$
\frac{\hbar^{2}}{2 m}\left(2 k_{1 z} q \cos \theta+q^{2} \cos ^{2} \theta\right)+\left|g_{n}\right| \mu_{\mathrm{B}} B_{\mathrm{r}}+\left(\zeta_{\mathrm{f}}-\zeta_{\mathrm{i}}\right) \hbar \omega_{\mathrm{c}}=E_{\mathrm{ph}}(q)+\hbar \omega_{\mathrm{rw}}
$$

The condition for spin-phonon resonance with external constant magnetic field strength $B_{\mathrm{r}}$ considering the equal probability of all possible angles $0<\theta<\pi$ from Eq. (3) with averaging over angles $\theta$ is written:

$$
\frac{\hbar^{2} q^{2}}{4 m}+\left|g_{n}\right| \mu_{\mathrm{B}} B_{\mathrm{r}}+\left(\zeta_{\mathrm{f}}-\zeta_{\mathrm{i}}\right) \hbar \omega_{\mathrm{c}}=E_{\mathrm{ph}}(q)+\hbar \omega_{\mathrm{rw}},
$$

where $g_{n}$ and $\omega_{\mathrm{c}}=e B_{\mathrm{r}} / m$ are the $g$-factor and cyclotron frequency depending on the $c$-band electron concentration; $\zeta_{\mathrm{f}}-\zeta_{\mathrm{i}}=1,2,3, \ldots$, differences of Landau level numbers (fitting parameter); and $E_{\mathrm{ph}}(q)$, energy of a phonon with wave vector $q$.

Let us examine separately absorption of acoustic and optical phonons.

Absorption of acoustic phonons. The energy of an acoustic phonon $E_{\mathrm{ph}}(q)=E_{\mathrm{ph}}\left(q_{\mathrm{a}}\right)=\hbar v_{\mathrm{a}} q_{\mathrm{a}}$, where $v_{\mathrm{a}}$ is the velocity of sound in the crystal $\left(v_{\mathrm{a}}=3.41 \cdot 10^{5} \mathrm{~cm} / \mathrm{s}\right.$ for InSb). According to Eq. (4), the magnetic field $B_{\mathrm{r}}$ reaches a maximum for $q_{\mathrm{a}}=2 m v_{\mathrm{a}} / \hbar$. The maximum $B_{\mathrm{r}}^{\max }=0.38 \mathrm{mT}$ is reached for $\zeta_{\mathrm{f}}-\zeta_{\mathrm{i}}=0$ for $n$-InSb with electron concentration $n=6 \cdot 10^{15} \mathrm{~cm}^{-3}$. The quantity $B_{\mathrm{r}}^{\max }=3 \mathrm{mT}$ for $n$-InSb with $n=5 \cdot 10^{18} \mathrm{~cm}^{-3}$. The $B_{\mathrm{r}}$ values calculated using Eq. (4) are much less than those observed (Fig. 1b and Table 1). Thus, absorption of acoustic phonons was not the reason for the magnetic resonance observed before [12].

Absorption of optical phonons. A transition of a $c$-band electron between Landau levels with spin inversion is possible upon absorption of a transverse optical phonon [24-30 and references therein]. According to the literature [4], the 


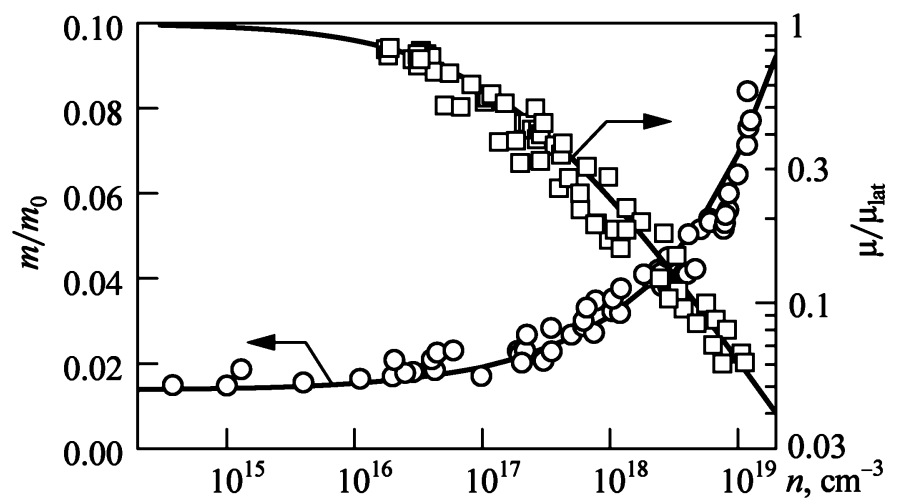

Fig. 3. Dependences of effective mass $\left(\mathrm{m} / \mathrm{m}_{0}\right)$ and mobility $\left(\mu / \mu_{\text {lat }}\right)$ of $c$-band electrons on their concentration at $300 \mathrm{~K}$; experimental values were taken from the literature $[4,33]$; lines are approximations using Eqs. (5) and (6).

energy of an optical phonon in $n$-InSb:Te crystals is $E_{\mathrm{ph}}(q)=E_{\mathrm{ph}}\left(q_{\mathrm{o}}\right)=22.9 \mathrm{meV}$ and depends weakly on $q_{\mathrm{o}}$ as compared to the term $\hbar^{2} q_{0}^{2} / 4 m$. The energy of an optical phonon in InSb crystals is of the order of that of thermal motion $(\sim 25.85 \mathrm{meV})$ at room temperature, i.e., the sample temperature during the magnetic resonance measurements. Then, only transverse optical phonons with wave vector $q_{\mathrm{o}}=0$ (center of the Brillouin zone) were considered for simplicity in calculating $B_{\mathrm{r}}$ using Eq. (4). Hence, optical phonons with wave vector $q_{\mathrm{o}} \neq 0$ but still satisfying the resonance condition of Eq. (4) for various $B_{\mathrm{r}}$ could contribute to the resonance line shape. The greater $q_{\mathrm{o}}$ was, the smaller $B_{\mathrm{r}}$ was. As a result, the resonance lines [transfer of $Y^{\prime}(B)$ line intensity from the low-field tail $B<B_{\mathrm{r}}$ into the high-field part $B>B_{\mathrm{r}}$ ] were slightly asymmetric in samples Nos. 1 and 2 and partially 3. Table 1 presents experimental $B_{\mathrm{r}}^{(\mathrm{e})}$ and $B_{\mathrm{r}}^{(\mathrm{t})}$ calculated using Eq. (4) upon absorption of optical phonons for discrete values of $\zeta_{f}-\zeta_{i}$ in the range from 16 (for sample No. 1) to 6 (No. 6). Equation (4) also shows that the greater frequency $\omega_{\mathrm{rw}}$ was, the greater $B_{\mathrm{r}}$ would be (under otherwise equal conditions) when the center of the electron spinphonon magnetic resonance line was recorded. This was experimentally confirmed [11].

The dependence of the effective mass $m$ on the concentration of $c$-band electrons had to be taken into account to calculate the properties of the $n$-InSb crystals. The experimental $c$-band electron effective mass $[4,33]$ was approximated as (Fig. 3):

$$
m / m_{0}=0.0136\left[1+\left(n / n_{\mathrm{m}}\right)^{1 / 2}\right]
$$

where $m_{0}$ is the electron mass in a vacuum; $n$, electron concentration; and $n_{\mathrm{m}}=6 \cdot 10^{17} \mathrm{~cm}^{-3}$.

The Fermi energy $E_{\mathrm{F}}$ in the $c$-band of degenerate InSb crystals (samples Nos. 4-6) without a magnetic field can be estimated using the standard formula [34]:

$$
E_{\mathrm{F}}=\hbar^{2}\left(3 \pi^{2} n\right)^{2 / 3} / 2 m
$$

where the effective electron mass $m=m(n)$ is determined by Eq. (5). The Fermi energy for sample No. $4\left(n=1 \cdot 10^{17} \mathrm{~cm}^{-3}\right)$ was $E_{\mathrm{F}} \approx 53 \mathrm{meV}$, which was approximately twice the thermal energy $(\sim 26 \mathrm{meV})$ at room temperature.

The experimental data for Hall and drift mobility of electrons $\mu=\mu(n)$ at room temperature [33] was approximated as (Fig. 3):

$$
1 / \mu=\left(1 / \mu_{\text {lat }}\right)\left[1+\left(n / n_{\mu}\right)^{2 / 3}\right],
$$

where $\mu_{\text {lat }}=9 \cdot 10^{4} \mathrm{~cm}^{2} / \mathrm{V} \cdot \mathrm{s} ; n_{\mu}=1.72 \cdot 10^{17} \mathrm{~cm}^{-3}$.

The experimental data $[2,35-37]$ for a $c$-band electron $g$-factor $g_{n}=g_{n}(n)$ at cryogenic temperatures was approximated as (Fig. 4, curve 1):

$$
g_{n}=g_{n 1}=a+b \log \left(n / \mathrm{cm}^{-3}\right)+c \log ^{2}\left(n / \mathrm{cm}^{-3}\right),
$$

where $a=191, b=-35.3$, and $c=1.283$.

According to the literature [38], the electron $g$-factor $g_{n}<0$ could be estimated from the formula: 


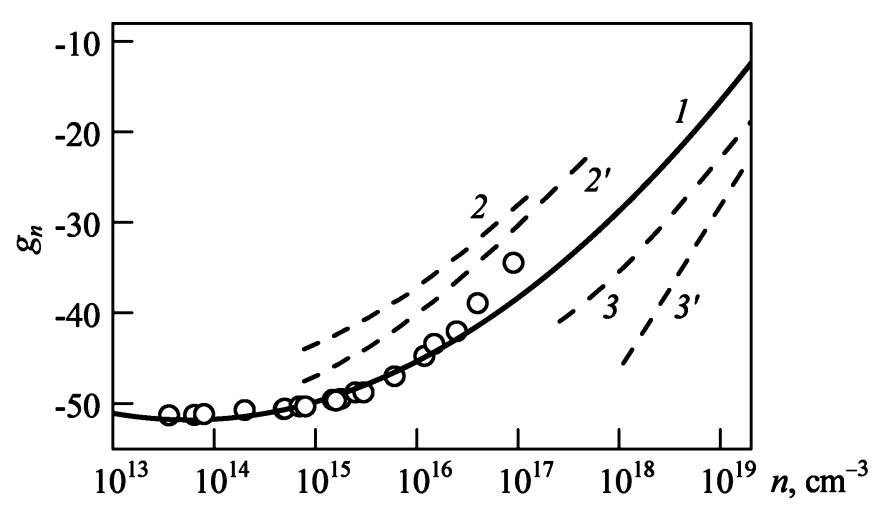

Fig. 4. Dependence of $g$-factor $g_{n}$ of $c$-band electron of InSb on electron concentration; experimental values were taken from the literature [2, 35-37]; approximation using Eq. (7) (1), approximations using Eq. (8) considering Eq. (5) for 4.2 and $300 \mathrm{~K}\left(2,2^{\prime}\right)$; approximations using Eq. (9) for 4.2 and $300 \mathrm{~K}\left(3,3^{\prime}\right)$.

$$
g_{n}=g_{n 2}=2\left[1-\left(\frac{m_{0}}{m}-1\right) \frac{\Delta_{2}}{3 E_{\mathrm{g} 2}+2 \Delta_{2}}\right],
$$

where $m=m(n)$ is given by Eq. (5); $\Delta_{2}=0.81 \mathrm{eV}$, the spin-orbit splitting of InSb valence band [4]; $E_{\mathrm{g} 2 \text {, bandgap energy }}$ $\left(E_{\mathrm{g} 2}=0.235 \mathrm{eV}\right.$ in InSb at $4.2 \mathrm{~K} ; 0.18 \mathrm{eV}$, at $\left.300 \mathrm{~K}\right)$.

According to the literature [39], the $g$-factor of a $c$-band electron with average kinetic energy $E_{\mathrm{av}}$ is:

$$
g_{n}=g_{n 3}=2\left[1-\frac{E_{\mathrm{p}}}{3}\left(\frac{1}{E_{\mathrm{g} 3}+E_{\mathrm{av}}}-\frac{1}{E_{\mathrm{g} 3}+\Delta_{3}+E_{\mathrm{av}}}\right)\right],
$$

where $E_{\mathrm{p}}=23.1 \mathrm{eV} ; E_{\mathrm{g} 3}=E_{\mathrm{g} 2} ; \Delta_{3}=0.803 \mathrm{eV} ; E_{\mathrm{av}}=3 k_{\mathrm{B}} T / 2$, the average electron energy in a nondegenerate (MaxwellBoltzmann) electron gas; $k_{\mathrm{B}}$, Boltzmann's constant; $E_{\mathrm{av}}=3 E_{\mathrm{F}} / 5$, the average energy of an electron with effective mass $m=m(n)$ in a degenerate (Fermi-Dirac) electron gas.

Figure 4 shows an approximation of the experimental data for a $c$-band electron $g$-factor from Eqs. (7)-(9).

Magnetic Resonance Integrated Intensity and Line Width. The magnetic component $B_{1} \cos \left(\omega_{\mathrm{rw}} t\right)$ of an electromagnetic wave under the experimental conditions [12] penetrated the sample from the ends (area $1.5 \times 2 \mathrm{~mm}^{2}$; Fig. 1a) to a depth equal to the skin-layer thickness. Therefore, only $c$-band electrons located within the skin-layer near the sample surface will contribute to the integrated intensity of magnetic resonance signal $Y[40,41]$.

The dependence of the specific electrical conductivity at constant current on the external magnetic field strength can be approximated as [10]:

$$
\sigma_{\mathrm{dc}}(B)=\sigma_{\mathrm{dc}} S(\mu B)=\frac{\sigma_{\mathrm{dc}}}{1+(\mu B)^{p}},
$$

where $\sigma_{\mathrm{dc}}=e n \mu(n)$ is the electrical conductivity at constant current without a magnetic field (Table 1$) ; \mu=\mu(n)$, the mobility of $c$-band electrons [Fig. 3; Eq. (6)]; $S(\mu B)=1 /\left[1+(\mu B)^{p}\right]$, a dimensionless function considering the decrease of electrical conductivity because of increased electron scattering rate in the bulk and on the surface of the samples, the Hall effect, and amplification of the electrical component of the RF field in the magnetized electron plasma with immobile positively charged donors and negatively charged acceptors, etc. [42-45]; and $p$, a fitting parameter ( $p=1$ was assumed in further calculations and was fully supported in experiments [12]).

The electrical conductivity of all examined $n$-InSb:Te samples according to the Drude model [46] without an external magnetic field was independent of angular frequency $\omega_{\mathrm{rw}}$ of the measuring signal, i.e., $\sigma\left(\omega_{\mathrm{rw}}\right)=\sigma_{\mathrm{dc}} /\left(1+\omega_{\mathrm{rw}} \tau\right) \approx \sigma_{\mathrm{dc}}$ where $\tau=\mu(n) m_{\sigma}(n) / e$ is the average relaxation time of the electron wave vector; $m_{\sigma}(n)=m(n)$, effective mass of the electrical conductivity (Fig. 3). In fact, $\omega_{\mathrm{rw}} \tau \approx 10^{-5}$ for $c$-band electron concentrations in the range from $n=6 \cdot 10^{15} \mathrm{~cm}^{-3}$ to $n=5 \cdot 10^{18} \mathrm{~cm}^{-3}$ so that $\sigma\left(\omega_{\mathrm{rw}}\right) \approx \sigma_{\mathrm{dc}}$. Then, $\omega_{\mathrm{c}} \tau=B_{\mathrm{r}} \mu(n) \approx 2$ for strength $B=B_{\mathrm{r}}$ (Table 1) considering Eq. (10). 
The characteristic penetration depth of a variable magnetic field $B_{1} \cos \left(\omega_{\mathrm{rw}} t\right)$ for a normal skin-effect into the studied sample (Fig. 1a) was defined as [40, 47]:

$$
\delta_{\mathrm{s}}(B)=\sqrt{\frac{2}{\omega_{\mathrm{rw}} \mu_{0} \sigma_{\mathrm{dc}}(B)}}=\delta_{\mathrm{s}} \sqrt{\frac{\sigma_{\mathrm{dc}}}{\sigma_{\mathrm{dc}}(B)}},
$$

where $\omega_{\mathrm{rw}}=2 \pi f_{\mathrm{rw}}=2 \pi \cdot 10^{7} \mathrm{rad} / \mathrm{s}$ is the angular frequency of the electromagnetic field of the measuring cavity coil; $\mu_{0}=1.257 \mu \mathrm{H} / \mathrm{m}$, the magnetic constant; $\sigma_{\mathrm{dc}}(B)$, specific sample electrical conductivity depending on constant magnetic field $B$ [Eq. (10)]; $\sigma_{\mathrm{dc}}=e n \mu(n)$, electrical conductivity for $B=0$. Mobility $\mu=\mu(n)$ was calculated using Eq. (6).

Let us average skin-layer depth $\delta_{\mathrm{s}}(B)$ over the scanning range of constant magnetic field strength $B$ in which the magnetic resonance line is recorded. The average skin-layer depth for the sample with number $j$ using Eqs. (11) and (10) for $p=1$ was calculated as

$$
\left\langle\delta_{\mathrm{s} j}\right\rangle=\frac{1}{3 B_{\mathrm{r} j}} \int_{0}^{3 B_{\mathrm{r} j}} \delta_{\mathrm{s} j}(B) \mathrm{d} B=\frac{\delta_{\mathrm{s} j}}{3 B_{\mathrm{r} j}} \int_{0}^{3 B_{\mathrm{r} j}} \sqrt{1+\mu_{j} B} \mathrm{~d} B,
$$

where $B_{\mathrm{r} j}$ is the resonant strength (i.e., the center of the resonance line; Table 1 ); $\delta_{\mathrm{s} j}=\sqrt{2 / \omega_{\mathrm{rw}} \mu_{0} \sigma_{\mathrm{dcj} j}}$, skin-layer depth without an external magnetic field (for $B=0$ ); $\mu_{j}=\mu_{j}(n)$, electron mobility in the $j$ th sample.

The integrated intensity of the magnetic resonance line of the $j$ th sample (original resonance line of radio wave absorption by the sample in a magnetic field) is

$$
Y_{j}=\int_{0}^{3 B_{\mathrm{r} j}}\left|Y_{j}^{\prime}(B)\right| \mathrm{d} B
$$

where $Y_{j}^{\prime}(B)=\mathrm{d} Q_{j} / \mathrm{d} B$ is the derivative of the cavity-coil quality factor $Q_{j}$ over the scanning magnetic field $\operatorname{strength} B$ during recording of the sample magnetic resonance (Fig. 1b).

According to Eq. (12), the number of electrons contributing to the magnetic resonance signal of the $j$ th sample with electron concentration $n_{j}$ and average skin-layer depth $\left\langle\delta_{\mathrm{s} j}\right\rangle$ is $n_{j}\left\langle\delta_{\mathrm{s} j}\right\rangle A$, where $A \approx 1.5 \times 2 \mathrm{~mm}^{2}$ is the area of the end surface of each of six samples (Fig. 1a). Then, the ratio of integrated intensities of the magnetic resonance signal of the $j$ th sample according to Eq. (13) to the signal intensity of sample No. 1 is equal to the ratio of the numbers of electrons in regions of their resonant absorption of the radio-wave magnetic component:

$$
\frac{Y_{j}}{Y_{1}}=\frac{n_{j}}{n_{1}} \frac{\left\langle\delta_{\mathrm{sj}}\right\rangle}{\left\langle\delta_{\mathrm{sl}}\right\rangle},
$$

where $j=1,2, \ldots, 6$ are the numbers of the $n$-InSb:Te samples.

Next, let us consider that the spin-phonon magnetic resonance signal width is mainly due to fluctuations of $c$-band electron potential energy because of their Coulombic interaction with the closest dopant ions and other $c$-band electrons. According to the literature $[48,49]$, the mean-square fluctuation of the electron electrostatic potential energy is

$$
W_{n} \approx 0.68 \frac{e^{2}}{4 \pi \varepsilon_{\mathrm{r}} \varepsilon_{0}}(1-K)^{1 / 6} n^{1 / 3},
$$

where $\varepsilon_{\mathrm{r}}=17.2$ is the relative dielectric permittivity of the InSb crystal matrix at room temperature [5]; $\varepsilon_{0}=8.854 \mathrm{pF} / \mathrm{m}$, an electrical constant; $n=(1-K) / N$, the $c$-band electron concentration; $N$, the concentration of Te hydrogen-like donor atoms, which exist totally in the +1 charge state; $K N$, the acceptor concentration (charge state -1$) ;(1+K) N$, the dopant ion concentration; $K \approx 0.1$, the degree of compensation of samples Nos. $1-6$.

Parameter $\zeta_{\mathrm{f}}-\zeta_{\mathrm{i}}=1,2,3, \ldots$ in Eq. (4) is a discrete value in the absence of electron potential energy fluctuations. The Landau levels in the crystalline sample are inequivalent [50] because of electrostatic fluctuations of the electron potential energy. Therefore, parameter $\zeta_{\mathrm{f}}-\zeta_{\mathrm{i}} \geq 1$ obtained by harmonizing the experimental $B_{\mathrm{r}}$ and those calculated using Eq. (4) can be approximated by a continuous variable depending on the electron concentration $n$ at $300 \mathrm{~K}$ :

$$
\frac{1}{\zeta_{\mathrm{f}}-\zeta_{\mathrm{i}}}=-0.604+0.0418 \log \left(n / \mathrm{cm}^{-3}\right) \text {. }
$$




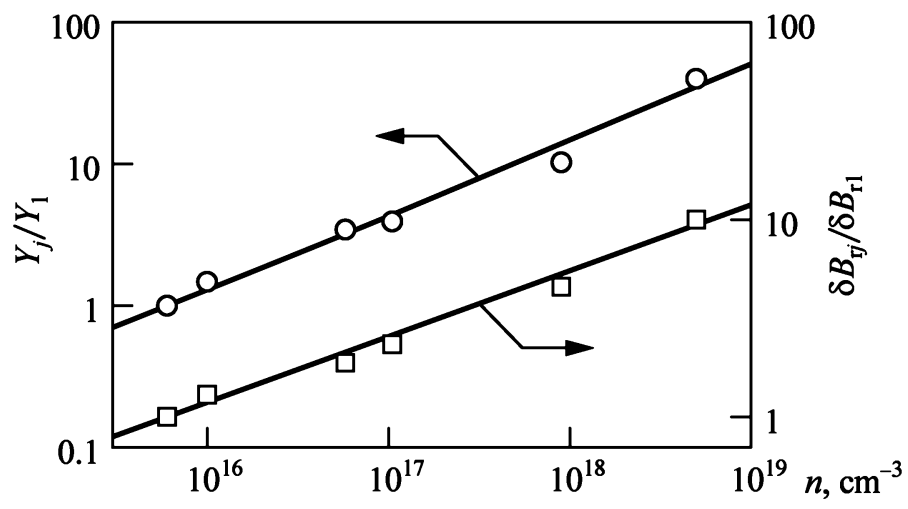

Fig. 5. Dependences of intensity ratio of magnetic resonance signals $\left(Y_{j} / Y_{1}\right)$ and line width ratio (from peak to peak) $\left(\delta B_{\mathrm{r} j} / \delta B_{\mathrm{r} 1}\right)$ in the $j$ th sample and in sample No. 1 on concentration $n$ of $c$-band electrons using Eqs. (14) and (17); points are experimental (Table 1).

The fluctuations of $c$-band electron potential energy cause the coexistence of vertical and nonvertical electron transitions on the single-electron-energy-spatial-coordinate diagram [51]. This condition determines the spin-phonon magnetic resonance linewidth during electron transitions between Zeeman sublevels of Landau levels. Then, the ratio of the linewidth (from peak to peak) in the $j$ th sample $\delta B_{\mathrm{rj}}$ to linewidth $\delta B_{\mathrm{r} 1}$ in sample No. 1 calculated from Eq. (15) is

$$
\frac{\delta B_{\mathrm{r} j}}{\delta B_{\mathrm{r} 1}} \approx \frac{W_{n j}}{W_{n 1}} .
$$

Figure 5 shows ratios of experimental intensities $Y_{j} / Y_{1}$ and widths (from peak to peak) $\delta B_{\mathrm{r} j} / \delta B_{\mathrm{r} 1}$ of magnetic resonance lines for sample $j$ to sample 1 (Table 1 gives the sample parameters).

Table 1 and Fig. 5 show that the ratio of magnetic resonance signal widths for samples with $j=1,2, \ldots, 6$ to the width of the signal for the first $(j=1)$ sample $\delta B_{\mathrm{r} j}^{(\mathrm{e})} / \delta B_{\mathrm{rl}}^{(\mathrm{e})}$ is practically the same as the ratio of the mean-square fluctuations of the $c$-band electron potential energy for the $j$ th and first samples $W_{n j} / W_{n 1}$.

Conclusions. A model for spin-phonon magnetic resonance in $n$-InSb:Te crystals at room temperature (resonance absorption of radio waves of frequency $10 \mathrm{MHz}$ at constant magnetic field) was proposed for the first time. Spin-phonon resonance in $n$-InSb samples with $c$-band electron concentrations from $6 \cdot 10^{15}$ to $5 \cdot 10^{18} \mathrm{~cm}^{-3}$ was calculated. The position of the magnetic resonance signal line center was related to the magnetic field strength required to produce that splitting of the $c$-band electron energy levels at which an electron transition was energetically possible. The transition occurred from a Zeeman sublevel of a lower Landau level (parabola) into one of the Zeeman sublevels of an upper Landau level with electron spin inversion via absorption by it of a transverse optical phonon of energy $22.9 \mathrm{meV}$. Radio waves of frequency $10 \mathrm{MHz}$ that detected magnetic resonance were also absorbed. The resonance line width (from peak to peak) was due to $c$-band electron potential energy fluctuations because of Coulombic interaction of them with dopant ions and among themselves. The signal integrated intensity was related to the dependence of the skin-layer thickness at $10 \mathrm{MHz}$ to the sample electrical conductivity at constant current in a magnetic field. The angular dependence of the radio-wave absorption resonance line in a magnetic field was determined by the anisotropy of the $c$-band electron $g$-factor. A quantitative description of known experiments that were not previously interpreted was given.

Acknowledgments. The work was supported by the State Scientific Research Program "Convergence-2020" of the Republic of Belarus, Belarusian Republic Foundation for Basic Research (Grant No. F19RM-054) and the European Union Framework Program for Development of Scientific Research and Technology Horizon2020 (Grant No. H2020-MSCARISE-2019-871284 SSHARE). One of us (NAP) is grateful to R. V. Parfen'ev and E. L. Ivchenko for constructive discussion of the article.

\section{REFERENCES}

1. G. A. Khodaparast, R. C. Meyer, X. H. Zhang, T. Kasturiarachchi, R. E. Doezema, S. J. Chung, N. Goel, M. B. Santos, and Y. J. Wang, Phys. E, 20, Nos. 3-4, 386-391 (2004). 
2. G. Bemski, Phys. Rev. Lett., 4, No. 2, 62-64 (1960).

3. M. A. Khayer and R. K. Lake, IEEE Trans. Electron Devices, 55, No. 11, 2939-2945 (2008).

4. O. Madelung, Semiconductors: Data Handbook, Springer, Berlin (2004).

5. S. Adachi, Properties of Semiconductor Alloys: Group-IV, III-V and II-VI Semiconductors, Wiley, Chippenham (2009).

6. Yu. M. Gal'perin, E. M. Gershenzon, I. L. Drichko, and L. B. Litvak-Gorskaya, Fiz. Tverd. Tela, 24, No. 1, 3-24 (1990).

7. E. O. Kane, J. Phys. Chem. Solids, 1, No. 4, 249-261 (1957).

8. E. Sijercic, K. Mueller, and B. Pejcinovic, Solid-State Electron., 49, No. 8, 1414-1421 (2005).

9. H. Kosaka, A. A. Kiselev, F. A. Baron, K. W. Kim, and E. Yablonovitch, Electron. Lett., 37, No. 7, 464-465 (2001).

10. R. S. Popovic, Hall Effect Devices, IOP Publishing, Bristol (2003).

11. M. V. Kondrat'ev, Fiz. Tverd. Tela, 19, No. 2, 616-617 (1977).

12. M. V. Kondrat'ev, Fiz. Tekh. Poluprovodn., 20, No. 8, 1485-1487 (1986).

13. N. A. Poklonskii, S. A. Vyrko, and A. G. Zabrodskii, Fiz. Tverd. Tela, 46, No. 6, 1071-1075 (2004).

14. N. B. Brandt and S. M. Chudinov, Usp. Fiz. Nauk, 137, No. 3, 479-499 (1982).

15. Y.-F. Chen, M. Dobrowolska, and J. K. Furdyna, Phys. Rev. B: Condens. Matter Mater. Phys., 31, No. 12, 7989-7994 (1985).

16. F. Qu, J. van Veen, F. K. de Vries, A. J. A. Beukman, M. Wimmer, W. Yi, A. A. Kiselev, B.-M. Nguyen, M. Sokolich, M. J. Manfra, F. Nichele, C. M. Marcus, and L. P. Kouwenhoven, Nano Lett., 16, No. 12, 7509-7513 (2016).

17. M. V. Kondrat'ev, Fiz. Tekh. Poluprovodn., 13, No. 2, 382-384 (1979).

18. A. V. Kessenikh, Usp. Fiz. Nauk, 179, No. 7, 737-764 (2009).

19. V. V. Slyn'ko, E. I. Slyn'ko, A. G. Khandozhko, and Yu. K. Vygranenko, Fiz. Tekh. Poluprovodn., 31, No. 10, 1187-1191 (1997).

20. P. Braun and S. Grande, Phys. Status Solidi B, 72, No. 1, K73-K76 (1975).

21. G. Dresselhaus, A. F. Kip, and C. Kittel, Phys. Rev., 98, No. 2, 368-384 (1955).

22. E. I. Rashba, Usp. Fiz. Nauk, 84, No. 4, 557-578 (1964).

23. W. Zawadzki, Phys. Lett., 4, No. 3, 190-191 (1963).

24. F. G. Bass and I. B. Levinson, Zh. Eksp. Teor. Fiz., 49, No. 3 (9), 914-924 (1965).

25. A. Yu. Matulis, Fiz. Tverd. Tela, 9, No. 8, 2238-2241 (1967).

26. S. T. Pavlov and Yu. A. Firsov, Zh. Eksp. Teor. Fiz., 49, No. 5 (11), 1664-1680 (1965).

27. S. T. Pavlov and Yu. A. Firsov, Fiz. Tverd. Tela, 7, No. 9, 2634-2647 (1965).

28. V. A. Margulis, Fiz. Tverd. Tela, 23, No. 3, 897-899 (1981).

29. R. V. Parfen'ev, G. I. Kharus, I. M. Tsidil'kovskii, and S. S. Shalyt, Usp. Fiz. Nauk, 112, No. 1, 3-36 (1974).

30. Yu. A. Firsov, V. L. Gurevich, R. V. Parfeniev, and I. M. Tsidil'kovskii, in: Landau Level Spectroscopy (Modern Problems in Condensed Matter Science, 27.2), G. Landwehr and E. I. Rashba (Eds.), North-Holland, Amsterdam (1991), pp. 1181-1302.

31. M. I. Kaganov and S. Klyama, Fiz. Tverd. Tela, 20, No. 8, 2360-2368 (1978).

32. V. I. Okulov, E. A. Pamyatnykh, and G. A. Al'shanskii, Fiz. Nizk. Temp., 35, No. 2, 194-196 (2009).

33. W. Zawadzki, Adv. Phys., 23, No. 3, 435-522 (1974).

34. K. W. Boer and U. W. Pohl, Semiconductor Physics, Springer, Berlin (2018).

35. R. A. Isaacson, Phys. Rev., 169, No. 2, 312-314 (1968).

36. A. E. Stephens, D. G. Seiler, J. R. Sybert, and H. J. Mackey, Phys. Rev. B: Solid State, 11, No. 12, 4999-5001 (1975).

37. A. V. Vdovin and E. M. Skok, Phys. Status Solidi B, 136, No. 2, 603-613 (1986).

38. L. M. Roth, B. Lax, and S. Zwerdling, Phys. Rev., 114, No. 1, 90-104 (1959).

39. K. L. Litvinenko, L. Nikzad, C. R. Pidgeon, J. Allam, L. F. Cohen, T. Ashley, M. Emeny, W. Zawadzki, and B. N. Murdin, Phys. Rev. B: Condens. Matter Mater. Phys., 77, No. 3, 033204(1-4) (2008).

40. J. Winter, Magnetic Resonance in Metals, Oxford University Press, New York (1971), 206 pp.

41. N. A. Poklonskii, S. A. Vyrko, O. N. Poklonskaya, N. M. Lapchuk, and S. Munkhtsetseg, Zh. Prikl. Spektrosk., 80, No. 3, 379-384 (2013) [N. A. Poklonski, S. A. Vyrko, O. N. Poklonskaya, N. M. Lapchuk, and S. Munkhtsetseg, J. Appl. Spectrosc., 80, 366-371 (2013)].

42. A. A. Ovchinnikov, Elektrokhimiya, 39, No. 1, 19-23 (2003).

43. A. V. Timofeev, Usp. Fiz. Nauk, 176, No. 11, 1227-1236 (2006).

44. V. V. Kosarev, N. A. Red'ko, and V. I. Belitskii, Zh. Eksp. Teor. Fiz., 100, No. 2 (8), 492-509 (1991). 
45. B. A. Aronzon and I. M. Tsidilkovskii, Phys. Status Solidi B, 157, No. 1, 17-59 (1990).

46. An. V. Vinogradov, Zh. Eksp. Teor. Fiz., 70, No. 3, 999-1008 (1976).

47. A. I. Veinger, A. G. Zabrodskii, T. V. Tisnek, and G. Biskupski, Fiz. Tekh. Poluprovodn., 32, No. 5, $557-563$ (1998).

48. N. A. Poklonski, S. A. Vyrko, A. I. Kovalev, and A. N. Dzeraviaha, J. Phys. Commun., 2, No. 1, 015013(1-14) (2018).

49. N. A. Poklonskii, S. A. Vyrko, and A. N. Derevyago, Zh. Belarus. Gos. Univ. Fiz., No. 2, 28-41 (2020).

50. L. B. Ioffe and A. I. Larkin, Zh. Eksp. Teor. Fiz., 81, No. 3 (9), 1048-1057 (1981).

51. N. A. Poklonskii and S. A. Vyrko, Zh. Prikl. Spektrosk., 69, No. 3, 375-382 (2002) [N. A. Poklonski and S. A. Vyrko, J. Appl. Spectrosc., 69, 434-443 (2002)]. 